\section{Prevalencia de enteroparasitosis en niños que asisten a una Unidad Educativa}

\author{
Prevalence of enteroparasitosis in children attending an \\ Educational Unit
}

\section{Prevalência de enteroparasitose em crianças atendidas em uma Unidade Educacional}

\author{
Milena Ruth Villca Mamani \\ milenavillca@hotmail.com
}

Recibido enero 2019 / Revisión febrero 2019 / Aceptado 1 de mayo 2019

\section{RESUMEN}

Introducción: Los parásitos intestinales son un problema de alta prevalencia entre los niños, especialmente en países en desarrollo, afecta a todas las edades y sexos, pero se presentan sobre todo en los primeros años de vida. Objetivo: Conocer la frecuencia de parasitosis intestinal en niños de 4 a 9 años, en la Unidad Educativa "Tcnl. Rafael Pabón" de la Comunidad de Tres Ríos en el último trimestre de la gestión 2018. Material y métodos: Investigación descriptiva y de campo, población de 36 niños. El examen fue coproparasitológico directo y el método de concentración Ritchie modificado. Resultados: La frecuencia de parasitosis fue de 69\%. El 100\% de las mismas por protozoarios, siendo los niños de 8 años los más afectados (32\%), el género masculino representa el $39 \%$ de los casos positivos. Los parásitos que se encontraron con mayor frecuencia son: entamoeba coli y endolimax nana. Conclusiones: La prevalencia de infección por parásitos intestinales en los niños es elevada. De 36 casos, se tiene 25 casos positivos resultando un $69 \%$ y 11 casos negativos correspondientes a un $31 \%$ de la población. Los niños de 8 años mostraron mayor frecuencia de parasitosis con un porcentaje de $32 \%$.

Palabras clave: parásitos; enfermedades parasitarias; parasitosis

\begin{abstract}
Introduction: Intestinal parasites are a highly prevalent problem among children, especially in developing countries, affecting all ages and sexes, but they occur especially in the first years of life. Objective: To know the frequency of intestinal parasitosis in children from 4 to 9 years old, in the Educational Unit "Tcnl. Rafael Pabón" from the Tres Ríos Community in the last quarter of the 2018 term. Material and methods: Descriptive and field research, population of 36 children. The examination was direct coproparasitological and the modified Ritchie concentration method. Results: The frequency of parasitosis was $69 \%$. $100 \%$ of them by protozoa, with 8 -year-old boys being the most affected $(32 \%)$, the male gender represents $39 \%$ of positive cases. The most frequently found parasites are: entamoeba coli and endolimax nana. Conclusions: The prevalence of intestinal parasite infection in children is high. Out of 36 cases, there are 25 positive cases resulting in $69 \%$ and 11 negative cases corresponding to $31 \%$ of the population. The 8 -year-old children showed a higher frequency of parasitosis with a percentage of $32 \%$.
\end{abstract}

Key words: parasites; parasitic diseases; parasitosis
MV: Universidad Mayor de San Andrés, Bolivia 


\section{RESUMO}

MV: Universidad Mayor de San Andrés, Bolivia
Introdução: Intestinal parasites are a highly prevalent problem among children, especially in developing countries, affecting all ages and sexes, but they occur especially in the first years of life. Objective: To know the frequency of intestinal parasitosis in children from 4 to 9 years old, in the Educational Unit "Tcnl. Rafael Pabón "from the Tres Ríos Community in the last quarter of the 2018 term. Material and methods: Descriptive and field research, population of 36 children. The examination was direct coproparasitological and the modified Ritchie concentration method. Results: The frequency of parasitosis was $69 \%$. $100 \%$ of them by protozoa, with 8-year-old boys being the most affected (32\%), the male gender represents $39 \%$ of positive cases. The most frequently found parasites are: entamoeba coli and endolimax nana. Conclusions: The prevalence of intestinal parasite infection in children is high. Out of 36 cases, there are 25 positive cases resulting in $69 \%$ and 11 negative cases corresponding to $31 \%$ of the population. The 8 -year-old children showed a higher frequency of parasitosis with a percentage of $32 \%$.

Palavras-chave: parasitas; doenças parasitárias; parasitoses

\section{INTRODUCCIÓN}

$\mathrm{L}$ os parásitos intestinales son un problema de alta prevalencia entre los niños. En los países en desarrollo y a pesar de la baja letalidad, cada año hay un repunte de las tasas de morbimortalidad, especialmente en los niños menores de 5 años.

Las condiciones sanitarias deficientes presentes en las comunidades, afectan a personas de todas las edades y sexos, pero en particular, niños en los primeros años de vida, ya que este grupo de población aún no ha adquirido los hábitos higiénicos necesarios para prevenirlas y no se ha desarrollado inmunidad frente a los diferentes tipos de parásitos.

Las parasitosis se encuentran entre las enfermedades y problemas de salud, a cuyo diagnóstico y tratamiento contribuye la estrategia Atención Integrada a las Enfermedades Prevalentes de la Infancia (AIEPI) de la Organización Panamericana de la Salud (OPS) y la Organización Mundial de la Salud (OMS), la cual sirve para identificar rápidamente las enfermedades y problemas de salud que ponen en riesgo al niño, aplicar medidas de prevención y promoción de la salud y dar información y educación a la población con respecto a la atención y cuidado del niño en el hogar (1). La OMS estima, que más de 2 billones de personas en el mundo, viven con enfermedades debido a los parásitos intestinales especialmente, en países en desarrollo. La OPS/OMS calcula que 20$30 \%$ de todos los latinoamericanos están infectados por helmintos intestinales (parásitos intestinales), mientras que las cifras en los barrios pobres, alcanzan con frecuencia el $50 \%$ y hasta el $95 \%$ en algunos grupos indígenas (PAHO) (2).

Las parasitosis son conocidas como enfermedades desatendidas, por la poca importancia que dan los gobiernos y por ser consideradas como baja prioridad de salud pública internacional (2). La mayor frecuencia de estas enfermedades enteroparasitarias se observa en los sectores rurales, por las condiciones de vida para el individuo.

Los parásitos pueden agruparse en dos grupos, protozoarios, tales como Entamoeba histolytica/E. dispar, Giardia lamblia, Criptosporidium sp y Metazoarios como Ascaris lumbricoides, Tenias, Trichuris trichiura, Enterobius vermicularis y Uncinarias. El mecanismo de transmisión 
varía dependiendo de cada parásito, sin embargo, la mayoría se adquiere al ingerir agua o alimentos contaminados, al consumir carne mal cocida, al no lavar las manos antes de comer o después de ir al baño, es decir que la vía de infección es ano-mano-boca (1).

Los síntomas producidos por los parásitos, dependen del organismo causante y de la condición del huésped, se presentan manifestaciones gastrointestinales como diarrea, dolor abdominal y distensión abdominal, y los Metazoarios producen afecciones en otros órganos o sistemas, entre ellos debilidad, palidez, pérdida de peso, anemia, tos crónica, prurito anal, sangreen heces e incluso la expulsión de vermes en las heces (3).

El diagnóstico de la parasitosis se logra mediante el estudio de las heces, llegando a visualizarse algunas formas evolutivas tales como quistes, ooquistes, huevos o larvas que ocasionalmente, pueden observarse directamente en las heces o en el margen del ano.

En Bolivia, existen aproximadamente 17 especies de parásitos como productores potenciales de infección intestinal humana, dentro de estas especies existen 5 protozoarios y 12 helmintos (4).

Estudios en comunidades indígenas, han demostrado que existe un cambio en los tipos de parásitos causales de enfermedad, probablemente por cambios de hábitos. Las comunidades indígenas que no pueden mantener sus costumbres y que sufren de pobreza, tienen mayores problemas de salud (5).

Una de los problemas más graves causados por enteroparasitosis es que esta puede interferir en la absorción de nutrientes provocando la mal nutrición proteína-energética y anemia ferropénica, que constituye un problema de salud en zonas rurales y urbanas (6).

El parasitismo intestinal es determinado por el acceso de las poblaciones a recursos materiales (posesión de bienes, calidad de la vivienda), recursos humanos (educación) y de saneamiento (tipo de sanitario, fuente de consumo de agua), así como por las prácticas de cuidado materno (alimentación, prevención e higiene) (7).

En Bolivia, no existe información oficial sobre la situación actual de las enfermedades enteroparasitarias y la prevalencia de parásitos intestinales tanto en la ciudad como en comunidades rurales, por este motivo el presente trabajo, pretende hacer un aporte al conocimiento de la prevalencia de enteroparasitosis en niños de la comunidad de Tres Ríos en el Departamento de La Paz.

\section{MATERIALES Y MÉTODOS}

$\mathrm{S}$ e realizó una investigación descriptiva, de campo, de corte transversal y prospectivo (8). La población de estudio estuvo constituida por 36 niños y niñas de 4 a 9 años de edad que asisten a la Unidad Educativa Tcnl. Rafael Pabón. Se obtuvieron muestras de heces fecales recién emitidas, las cuales se recolectaron en frascos de boca ancha y tapa rosca $\mathrm{y}$ fueron directamente $\mathrm{y}$ enviadas a valoración por el Instituto Nacional de Laboratorios de Salud (INLASA), para su posterior interpretación, mediante la realización de un examen coproparasitológico simple y por concentración. Se informó a los padres y a los niños adecuadamente sobre la patología, la forma de infección y la prevención de la parasitosis, con este fin se explicó la importancia de llevar a cabo este estudio. Esta investigación cumple con los requisitos éticos pertinentes declarados en el Tratado de Helsinki, se solicitó el asentimiento para la toma de las muestras previa explicación clara de los alcances de la investigación. 


\section{RESULTADOS Y DISCUSIÓN}

$\mathrm{U}$

na vez obtenido los resultados de los exámenes efectuados, se procedió al análisis de los mismos:

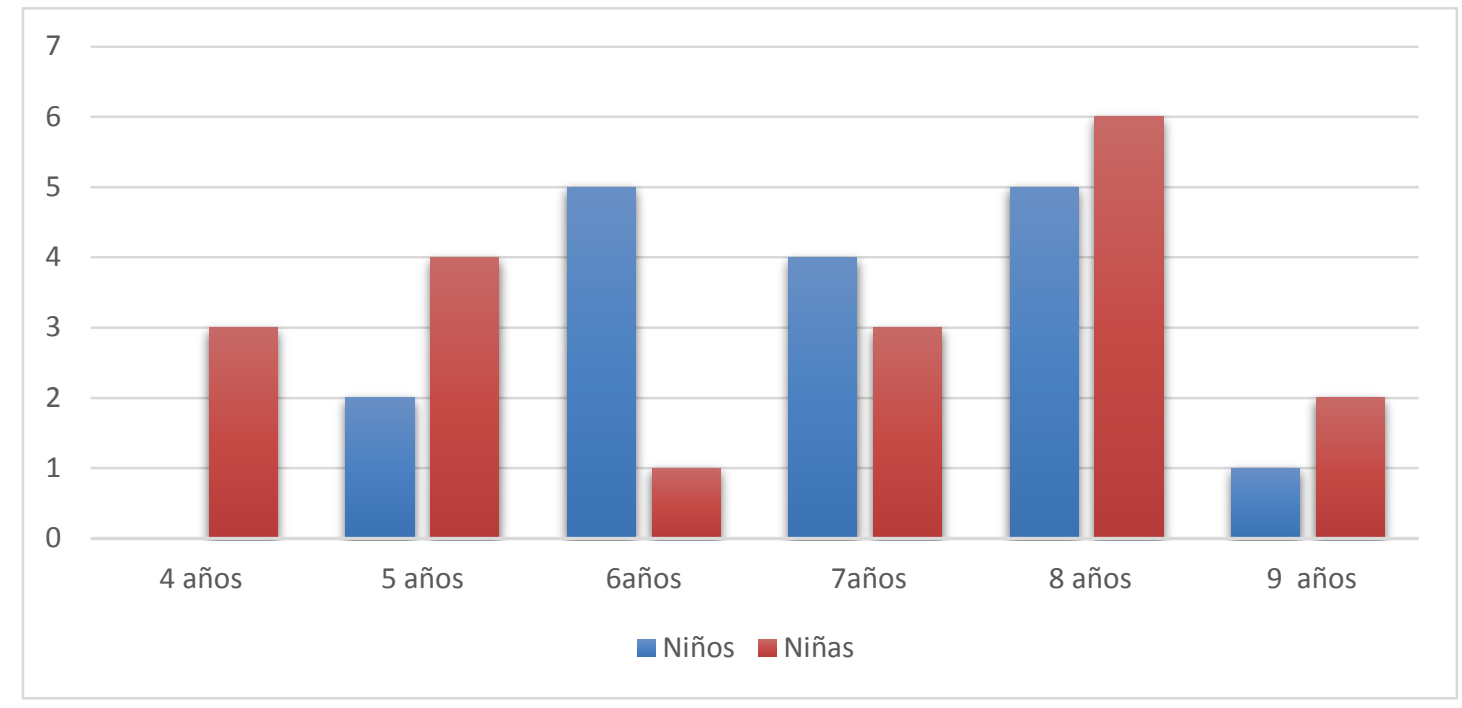

Gráfico 1. Distribución de niños por edad y sexo en frecuencia absoluta

Se evidencia que de la población evaluada, la mayoría corresponde a la edad de 8 años y seis años respectivamente

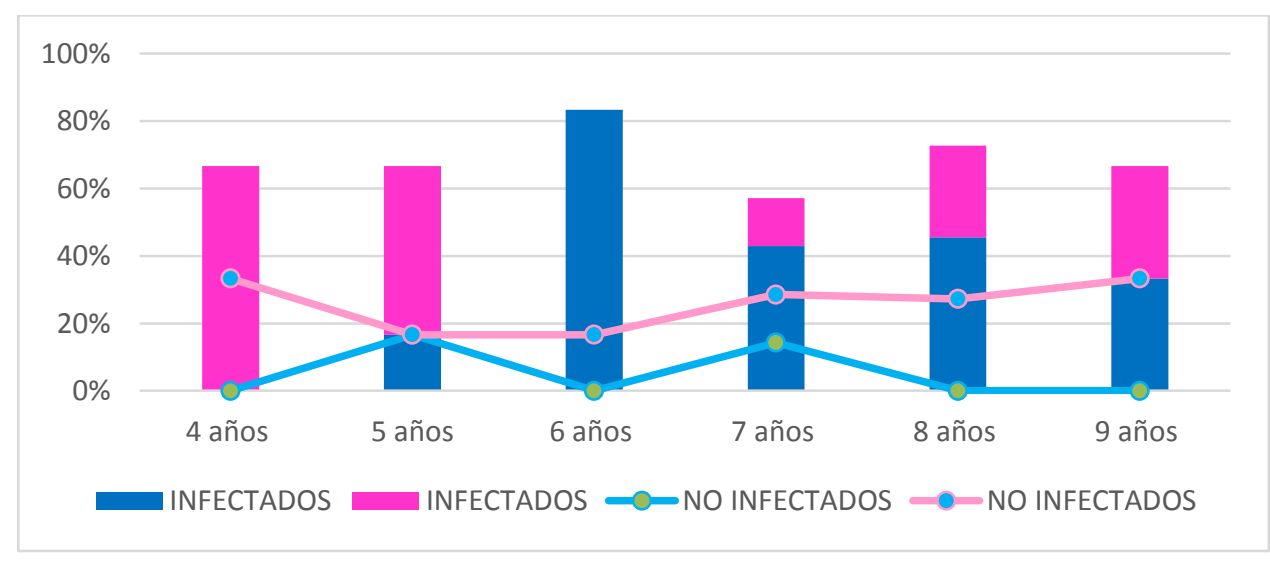

Gráfico 2. Relación población infectada y no infectada por edad y sexo

El gráfico muestra que hay mayor cantidad de mujeres no infectadas en todas las edades en relación a un 30\% aproximado del total de muestras, y más del 50\% en cada edad, son los varones con más tasas de infección parasitaria. 


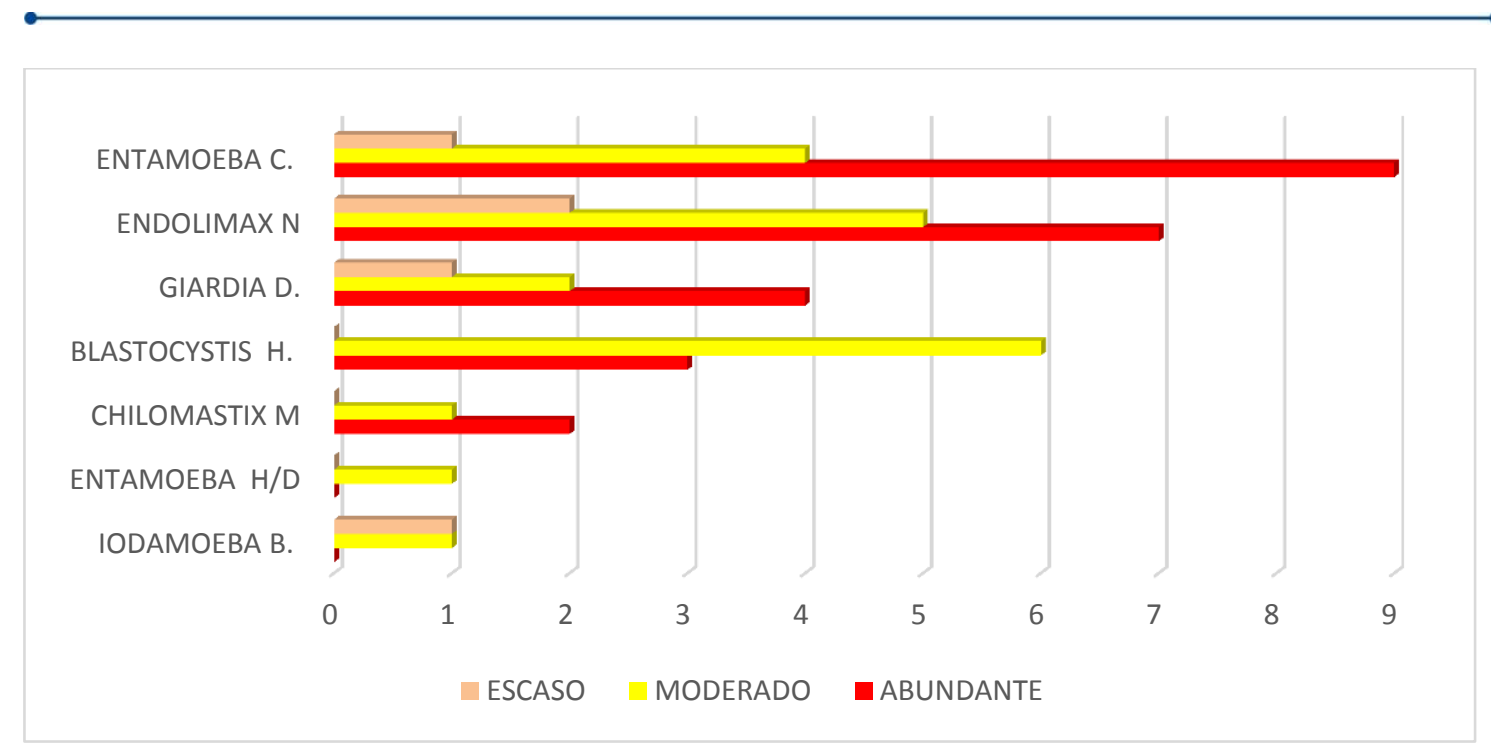

Gráfico 3. Parásitos detectados en muestras, relación a su tipo

Se encuentran 7 tipos de parásitos protozoarios presentes en las muestras de los niños estudiados, entre ellos Entamoeba coli y Endolimax nana quienes se encuentran en mayor cantidad, en los pacientes parasitados con presencia moderada y abundante, lo que representa $56 \%$ en ambos casos. El siguiente protozoario en mayor cantidad es Blastocistis hominis que representa un 36\%, le continúa Giardia lambdia 24\%, Chilomastix mesnilii 12\%, Lodamoeba butschlii $8 \%$ y finalmente se encuentra el complejo Entamoeba histolytica/dispar que representa al $4 \%$.

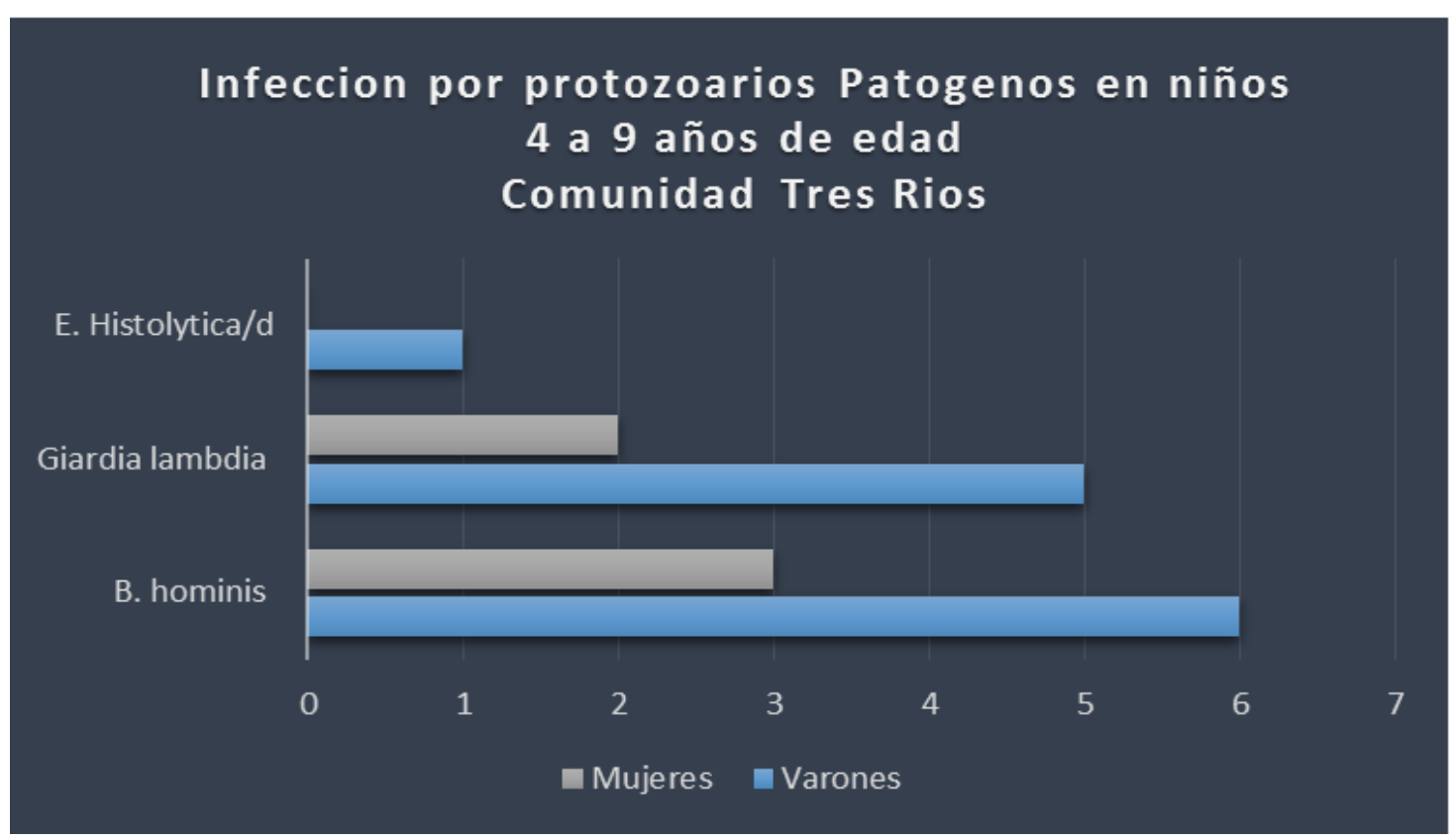

Gráfico 4. Parásitos patógenos detectados en las muestras estudiadas por sexo

En el gráfico 4, se evidencia la presencia los parásitos protozoarios encontrados en los resultados del estudio coproparasitológico. La frecuencia de infección por protozoarios patógenos en niños de 4 a 9 años, donde se halla que Blastocystis hominis se encuentra parasitando a 6 varones que corresponde a $43 \%$ y a 3 mujeres que representan el $27 \%$. 


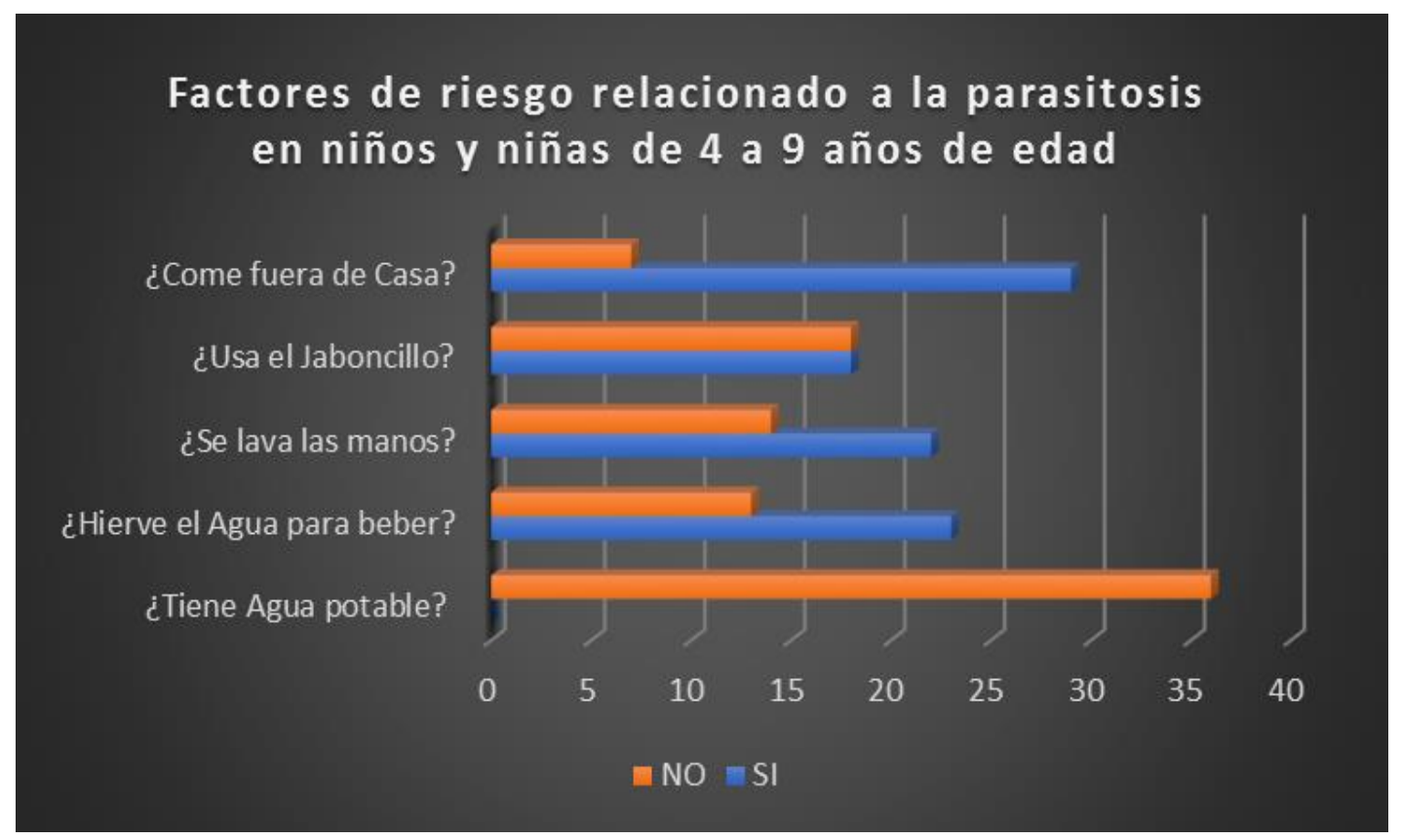

Gráfico 5. Factores relacionados con parasitosis en la población estudiada

En el presente cuadro, se observan factores de riesgo relacionados con el parasitismo en la población de estudio en general. Se puede evidenciar, que la totalidad de la población de estudio no posee agua potable. Además, que 29 niños y niñas comen fuera de casa a diferencia de 7 que no lo hacen. Llama la atención que en proporciones iguales, la mitad de los estudiados se lava las manos después de ir al baño usando jaboncillo. Un dato interesante, es que la mayoría de la población hierve el agua para beberla, que 23 personas de 36 y solo 13 de ellos, no lo hacen y finalmente, más de la mitad de los estudiados, se lava las manos constantemente. Los factores de riesgo más importantes para el parasitismo, están asociados a la carencia de servicio de agua potable.

\section{Discusión}

En Bolivia el predominio del parasitismo intestinal sigue siendo un importante problema de salud pública, por su alta prevalencia y distribución elevada. Este puede presentarse en forma endémica, aislada o en brotes esporádicos, ya que cada país tiene problemas diferentes de acuerdo a los factores condicionantes, demográficos, socioeconómicos, ambientales y sobre todo educacionales.

En el presente trabajo de un total de 36 muestras, el $69 \%$ son positivos y el $11 \%$ negativos. El grupo etario también es importante destacar, debido al que está en mayor peligro de parasitosis es de 8 a 9 años de edad con un $40 \%$ y que la mayor vulnerabilidad a la parasitosis está en el sexo masculino.

La prevalencia de infección por parásitos, está dada por protozoarios que llegaron a ser el 100\% y no existió la presencia de Helmintos, esto puede deberse a la influencia de los ecosistemas ya que en un informe Técnico del Ministerio de Salud del año 2000, indica que la Zona Andina no es propicia para el desarrollo del ciclo biológico de los parásitos, particularmente de los nematodos intestinales (5). 
Otro dato importante, es que los índices de pobreza y la población migrante favorecen al parasitismo intestinal, esto tiene una importante connotación ya que la comunidad de Tres Ríos aloja además de sus pobladores a personas que vienen de otros lugares a trabajar a los campamentos mineros que lo circundan (4).

La causa principal de parasitosis, se relaciona a la poca y mal higiene en muchos casos. La educación por parte de los padres de familia es muy escasa y la mayoría de las familias de la Comunidad, se dedica a la minería.

En los trabajos realizados se observa que el factor causante de parasitosis se debe al consumo de agua no potable, consumo de alimentos contaminados y sobre todo la poca higiene de los niños $(4,5)$.

\section{CONCLUSIONES}

$\mathrm{E}$ n conclusión, la parasitosis incidió más en el sexo masculino respecto al femenino llegando a ser un $36 \%$ en relación a toda la población de estudio. Al parecer la relación que existe entre la parasitosis encontrada y los factores de riesgo asociados a ella, en la población de estudio está relacionada a la carencia de servicios básicos tales como el agua potable, además esto se añade hábitos personales como la higiene y la alimentación fuera de casa que presentó la mayoría de los pacientes que formaron parte de este estudio. Se recomienda el desarrollo de nuevas investigaciones que ayuden a determinar la causa relacionada a los factores de riesgo que desencadenan la parasitosis no solamente en niños, sino también en la población en general.

- Conflicto de interés: el autor declara no presentar conflicto de interés.

- Financiamiento: autofinanciamiento

- Agradecimientos: Ninguno declarado por el autor.
- Investigación realizada bajo consentimiento informado, apegada a los tratados bioéticos

\section{REFERENCIAS BIBLIOGRÁFICAS}

1. Pan American Health Organization World Health Organization. First session of the subcommittee on program, budget, and administration of the executive committee. Washington. $2007 . \quad$ Disponible en: https://www.paho.org/english/gov/ce /spba/spba1-05c-e.pdf

2. Organización Mundial de la Salud y un conjunto de colaboradores dan a conocer un nuevo plan coordinado para tratar a millones de personas que sufren enfermedades tropicales desatendidas. [Internet].2006. Disponible en: https://www.who.int/mediacentre/news /releases/2006/pr60/es/index1.html

3. Cazorla-Perfetti D. Las enfermedades parasitarias intestinales como un problema de salud global. Invest. clin [Internet]. 2014 Mar [citado 201824 de febrero]; 55(1): 93-94. Disponible en: http://ve.scielo.org/scielo.php?script=s ci_arttext\&pid=S053551332014000100 010\&lng=es

4. Mollinedo P, Prieto B. El enteroparasitismo en Bolivia. Ministerio de Salud y Deporte, Dirección Nacional de Servicios de Salud, Programa Nacional del Escolar y Adolescente, Unidad de Parasitología: INLASA; 2006

5. Luna $S$, Jiménez $S$, López $R$, Soto $M$, Benefice E. Prevalencia de parasitismo intestinal en niños de comunidades indígenas del Rio Beni. Visión Científica. [Internet]. 2009 [citado 201824 de febrero]; 1(2): 37-46. Disponible en: http://www.revistasbolivianas.org.bo/ scielo.php?script=sci_arttext\&pid=S222 2-43612009000100007\&lng=es

6. Beaver, P. Cup, E. y Jung, R. Parasitología clínica de Craig y Faust. 3ra. ed. México: Masson; 2003

7. Alvarado B, Vásquez L. Determinantes sociales, prácticas de alimentación y consecuencias nutricionales del parasitismo intestinal en niños de $7 \mathrm{~A}$ 18 meses de edad, en Guapi, Cauca. 
Biomédica. 2016; 26:82-94. DOI: https://doi.org/10.7705/biomedica.v2 $6 \mathrm{i} 1.1397$
8. Hernández, R. Fernández, C. Baptista, MP. Metodología de la investigación. 5ta ed. México: McGraw-Hill; 2010 